EXPERIENCIAS EDUCATIVAS

\title{
LA ENSEÑANZA DE LA CREATIVIDAD EN ALUMNOS DE PSICOLOGÍA DE LA UNIVERSIDAD TECNOLÓGICA DE BOLÍVAR (CARTAGENA, COLOMBIA)
}

TEACHING OF CREATIVITY TO PSYCHOLOGY STUDENTS AT THE UNIVERSIDAD TECNOLÓGICA DE BOLÍVAR (CARTAGENA, COLOMBIA)

Pedro Vázquez-Miraz

Universidad Tecnológica de Bolívar 
RECIBIDO: $10 / 12 / 2020$

ACEPTADO: $10 / 03 / 2021$

\section{RESUMEN}

Se presenta en el siguiente trabajo la experiencia pedagógica del programa de psicología de la Universidad Tecnológica de Bolívar (UTB), una universidad privada de Colombia (Cartagena de Indias) sobre cómo este programa académico enseña a sus educandos el abstracto concepto de creatividad. Un constructo teórico fundamental para entender el desarrollo de las habilidades estudiantiles en el ámbito artístico y la resolución de problemas, entre otros factores. Los planes de curso de este centro académico se centran en la potenciación de una formación integral de los estudiantes y es por lo que todos ellos, además de las materias específicas de su carrera, cursan de manera obligatoria asignaturas de humanidades y de otras áreas. De este modo los estudiantes universitarios de psicología de la UTB adquieren los conocimientos sobre la creatividad desde tres perspectivas diferentes: 1. la perspectiva de la psicología cognitiva desde la materia de Cognición, 2. la visión de las asignaturas electivas de humanidades y 3. la postura económica desde el curso Creatividad y Emprendimiento. Gracias a este multidisciplinar pensum académico se permite al futuro psicólogo tener múltiples enfoques epistemológicos sobre la creatividad basados en diferentes métodos de enseñanza.

\section{PALABRAS CLAVE}

\section{Creatividad, Educación universitaria, Psicología cognitiva, Enseñanza}

\section{A B S T R ACT}

The following paper presents the pedagogical experience of the psychology program at the Universidad Tecnológica de Bolívar (UTB), a private university in Colombia (Cartagena de Indias) on how this academic program teaches its students the abstract concept of creativity. A fundamental theoretical construct to understand the development of student skills in the artistic field and problem solving, among other factors. The course plans of this academic center are focused on the promotion of a comprehensive training of students and that is why all of them, in addition to the specific subjects of their career, are compulsorily taking subjects from the humanities and other areas. In this way, UTB psychology students acquire knowledge about creativity from three different perspectives: 1. the perspective of cognitive psychology from the subject of Cognition, 2. the vision of the Humanities elective subjects and 3. the economic position from the Creativity and Entrepreneurship course. This multidisciplinary academic curriculum allows the future psychologist to have multiple epistemological approaches to creativity based on different teaching methods.

\section{KEYWORDS}




\section{INTRODUCCIÓN}

La creatividad es una característica del completo desarrollo de los seres humanos junto a otras facultades exclusivas, tales como la imaginación o la originalidad y que están asociadas intrínsicamente al concepto psicológico de autorrealización (Maslow, 1968), una característica innata del individuo que también es modificada por diferentes agentes sociales como la familia o el colegio (Rogers, 1975). Mas para Alonso (2016) la creatividad es una variable que en educación puede ser estimulada, motivada o desarrollada pero nunca enseñada pues el alumno llega al sistema educativo con ella dentro de su propio ser. Postura que no compartimos pues consideramos que la creatividad también puede ser impartida como un concepto teórico y práctico en el ámbito universitario, siendo ésta la motivación fundamental de este documento.

Una de las habilidades fundamentales que los maestros deben desarrollar en las aulas universitarias para que los estudiantes de estos centros educativos puedan adquirir los complejos conocimientos de las diferentes disciplinas académicas que se dictan en su seno es el de la creatividad (De la Torre y Violant, 2002), una expresión polivalente que ha sido comprendida de diversas maneras y desde distintas posturas del conocimiento humano y que en este trabajo se ha asumido de una condición integral (Huidobro, 2004, p. 124):

\footnotetext{
Concepto que ha surgido en la bibliografía por la necesidad de explicar la aparición de productos que suponen una transformación radical de un estado anterior, lo cual lleva a inferir la existencia de una persona que posee una constelación de rasgos intelectuales, de personalidad y motivacionales que le capacitan para utilizar la metacognición de un modo óptimo.
}

Dicha utilización óptima permite dar respuesta a un fallo o hueco en el conocimiento. Además, la persona ha de encontrarse inmersa en un contexto carente de obstáculos, que le presente modelos o parangones y le facilite los recursos necesarios.
La creatividad es un elemento esencial necesaria en el proceso educativo pues permite el desarrollo de aspectos cognitivos más relevantes para desempeño estudiantil (López-Martínez y Martín-Brufau, 2010). De este modo la incorporación de lo creativo a las instituciones educativas es una necesidad social para así conseguir una instrucción de mejor calidad (Martínez y Martín-Brufau, 2010), si bien este logro pedagógico no es tan fácil de conseguir. Como nos afirman Romo, Alfonso-Benlliure y Sánchez-Ruiz (2016, p. 94), "la necesidad de evaluar la creatividad reside en su relevancia social y educativa. Pero la creatividad es un proceso complejo y el planteamiento de su evaluación debe ser coherente con dicha complejidad". Es por ello por lo que, aunque toda la comunidad educativa está a favor de potencializar la creatividad, el hacerlo de un talante riguroso no es tan sencillo por la falta, entre otros aspectos, de conocimientos empíricos validados sobre esta temática (Soh, 2000), puesto que como comentan Elisondo, Donolo y Rinaudo (2009, p. 2):

La creatividad encuentra pocos detractores en los contextos educativos, difícilmente hallaremos algún investigador o docente que sostenga que ésta no es uno de los propósitos de la educación. Sin embargo, en muchas ocasiones, aparece sólo como un objetivo general que pocas veces logra concreciones en los contextos áulicos, en las planificaciones docentes y en las actividades pedagógicas diarias. Tal vez esta situación se deba a la dificultad intrínseca que supone proponer a la creatividad como propósito educativo, entre otras cosas, por la poca claridad existente acerca del término y por los desacuerdos vigentes respecto de los modos más apropiados de promoción de los procesos creativos.

Como docente de planta de una universidad, enseñar la creatividad y fomentarla en nuestro ámbito de trabajo es un loable objetivo didáctico del que dependen numerosos determinan- 
tes. Dentro de las múltiples variables existentes a la hora de realizar este proceso destacarían tres aspectos básicos: el tipo de docente y su estilo educativo (Soh, 2000), las características intrínsecas del estudiante universitario' (Snyder, Hammond, Grohman y Katz-Buonincontro, 2019) y en particular sus estilos de pensamiento (Zhu y Zhang, 201 1; Gutiérrez-Braojos, Salmeron-Vilchez, Martin-Romera y Salmerón-Pérez, 2013), y el propio proceso de enseñanza superior con todas sus virtudes (interdisciplinariedad...) y todos sus defectos (monotonía de las clases magistrales, la burocracia...) (Fleith, 2019). Sobre el primer elemento, cabe recalcar que los profesores, basándonos desde los principios de la teoría sociocultural de Vigotsky, al fin y al cabo, somos facilitadores del proceso de adquisición y elaboración de nuevos conocimientos por parte del alumnado, englobando también toda la información práctica y teórica relacionada con el aprendizaje de la creatividad (D'Angelo, 1999; Alessandroni, 2017).

Por consiguiente, consideramos que puede ser de interés real para la comunidad científica interesada en este tópico el conocer cómo en un programa de psicología de una universidad privada colombiana de Cartagena de Indias (Universidad Tecnológica de Bolívar, UTB) desde tres diferentes áreas del conocimiento humano (psicología, humanidades y economía) se explica la creatividad, enfatizándose así el componente pedagógico de la multidisciplinariedad.

\section{METODOLOGÍA}

La presente experiencia pedagógica que se relata en este informe se nutre en la recopilación de información académica de cuatro asignaturas de la Universidad Tecnológica de Bolívar (Cognición, Escritura creativa, Fotografía creativa, y Creatividad y emprendimiento) ligadas a la creatividad desde el análisis de sus respectivos sílabos. Un documento estructurado que abarca el conjunto de actividades académicas de una determinada materia y en el cual se definen detalladamente los resultados de aprendizaje, los objetivos, los contenidos, las metodologías y la evaluación a desarrollar

1 Para Kettler, Lamb, Willerson y Mullet (2018) induso los profesores que dicen valorar la creatividad en sus estudiantes pueden considerar que las genuinas caracteństicas de las personas creativas son indeseables en sus clases. por parte de los agentes educativos en base al modelo pedagógico de la institución educativa (Cedeño-Sempértegui, 2015).

\section{LA ENSEÑANZA DE LA CREATIVIDAD EN EL PROGRAMA DE PSICOLOGÍA DE LA UTB}

Dentro del modelo pedagógico que rige la UTB, uno de sus cinco principios básicos es la Innovación y la creatividad, siendo el deber de esta universidad la de plantear nuevas formas de hacer las cosas y propiciar la llegada de ellas. Actitudes necesarias tanto en el docente como en el estudiante para dar lugar a la autonomía, la creación y el desarrollo de los conocimientos y sus formas diversas de aplicación en contextos diversos.

Es por ello que en el presente documento se procura plasmar de forma escueta y resumida como se imparte la enseñanza de la creatividad en tres concretas clases de pregrado que un estudiante de psicología de la UTB puede cursar a lo largo de toda su carrera²: la materia opcional de psicología Cognición, la asignatura obligatoria de Creatividad y Emprendimiento y diferentes electivas relacionadas con el arte, considerándose estas últimas necesarias para la formación integral del estudiante, sea cual sea su carrera de grado, para así lograr la plena manifestación espiritual del ser humano (H. del Amo, 2016).

\section{La enseñanza de la creatividad en el programa de psicología de la UTB desde la psicología cognitiva}

La materia de Cognición es una asignatura semestral seleccionable del programa de psicología de la UTB, más específicamente del área de la Psicología Básica y de los Procesos Psicológicos ${ }^{3}$ (junto a otras asignaturas como Lenguaje, Aprendizaje, Sensación y percepción y Motivación y emoción) en la que el alumno al impartirla debe conocer las bases teóricas de

\footnotetext{
2 En todas las asignaturas de la UTB los estudiantes acceden al material académico por medio de elementos virtuales, siendo denominado el mecanismo que maneja esta institución SAMO (Sistema de Aprendizaje Virtual Interactivo); una plataforma de aprendizaje basada en contenido interactivo sobre Intemet.
}

3 El alumno de psicología de la UTB debe cursar tres materias de Procesos Psicológicos de las cinco que se ofertan. 
la comprensión del procesamiento de la información desde la perspectiva de la psicología cognitiva. Respecto a la metodología particular aplicada en esta materia, la clásica división entre clases prácticas y teóricas es un referente que siempre se mantiene durante todo el semestre académico. Así este curso está conformado por cuatro unidades temáticas bien diferenciadas:

1. Introducción a la psicología cognitiva y evolución histórica de la disciplina.

2. Atención y memoria como ejemplos de procesos psicológicos básicos.

3. Pensamiento y resolución de problemas como ejemplos de procesos psicológicos superiores.

4. El papel de la inteligencia en el ámbito de la cognición.

Es en el tercer punto de la asignatura (ya avanzado el curso) donde se introduce al estudiante conceptos relacionados con la creatividad, entendida ésta como un tipo de pensamiento creativo, sin embargo, como señalan Romo et al. (2016) la inteligencia (concepto dado en la última unidad temática) es un elemento explicativo fundamental para comprender esta noción, siendo dos de los vocablos asociados al intelecto más relevantes para tal efecto el de producción divergente ${ }^{4}$ y productos transformacionales ${ }^{5}$ (Romo, 1986). Términos (operaciones) originarios de la teoría clásica multifactorial de inteligencia de Guilford (1956).

En la ya mencionada tercera unidad, la creatividad se introduce de forma teórica (en dos únicas sesiones magistrales de dos horas cada una) dentro del tema denominado Psicología del Pensamiento que aborda a los procesos psicológicos superiores (ya explicados otros procesos psicológicos básicos tales como la atención y la memoria). Para exponer el término psicológico de creatividad a los alumnos se maneja en la clase como referente central

4 Constructo definido como "generación de altemativas lógicas a partir de una información dada, ayya importancia se halla en la variedad, cantidad y relevancia de la producción a partir de la misma fuente" (Romo, 1986, p. 181).

5 Concepto asumido como "cambios de diversas dases (redefiniciones, transposiciones, revisiones o modificaciones) en la información existente" (Romo, 1986, p. 181). la teoría de cuatro etapas (preparación, incubación, iluminación y verificación) de Wallas (1926). Una perspectiva teórica que mantiene intacto el interés científico en la comunidad académica actual (Sadler-Smith, 2015; Nuha, Waluya y Junaedi, 2018; Amabile, 2019).

Conforme al apartado práctico de la materia Cognición que versa sobre la creatividad, se le presenta a los estudiantes un único taller de este tema en el que después de la pertinente explicación introductoria se desarrolla, con los propios educandos, la propia actividad. Después, en horario extra-académico, ellos realizan esa misma labor con una muestra reducida que obtienen por conveniencia, debiendo entregar después de una semana un breve informe científico (10-15 páginas) con la típica estructura de este tipo de productos académicos ${ }^{6}$ con los datos que han recogido y su pertinente interpretación psicológica.

El material con el que se trabaja en este curso lo práctico de la creatividad es un popular acertijo denominado el rompecabezas de los nueve puntos (Jelle, 2017), en él los estudiantes tienen que unir nueve puntos (conformados éstos como si fueran un cuadrado imaginario) con cuatro líneas rectas sin levantar el útil de escritura del papel, siendo la única solución posible la de salirse de ese hipotético polígono ${ }^{7}$ (Figura 1), una forma de pensamiento creativo que en idioma inglés se conoce como thinking outside the box (Jelle, 2017). Un interrogante que se puede asociar al mundo de las matemáticas discretas, a la teoría de los grafos de Euler y al célebre problema de los siete puentes de Königsberg que resolvió este matemático en el siglo XVIII (Rosen, 2003).

6 Ese documento debe estar estructurado y poseer los siguientes apartados: Portada, Introducción, Metodología, Resultados, Discusión de Resultados y Referencias bibliográticas (Tamayo, 2004). Este informe es revisado por un sistema antiplagio (Tumitin $®$ ) para evitar posibles fraudes académicos. Una medida de seguridad que al igual que las evidencias encontradas por Dahl (2007) fue bien aceptada por los estudiantes universitarios, pero en varias ocasiones causando cierto malestar por la incertidumbre causada debido al desconocimiento de cómo se debe citar fuentes de otros autores Názquez-Mircz, Rentería, Marínez y Zapata, 2020).

7 Como requisito obligatorio antes de iniciar el taller, el docente siempre se asegura de que todos sus estudiantes desconocen el puzle en el que se basa esta actividad educativa. 


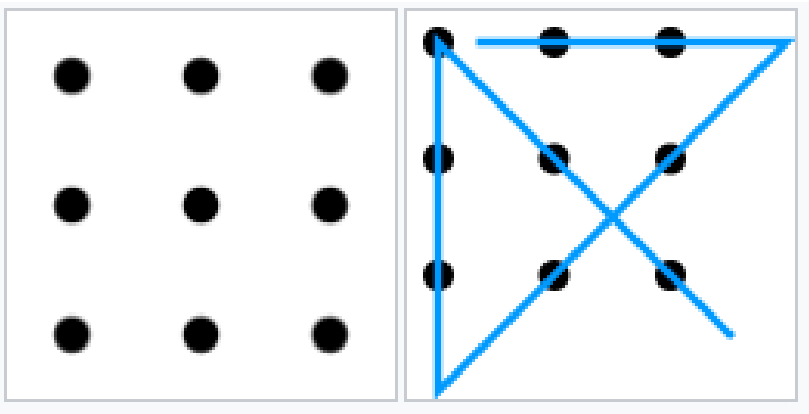

Figura 1. El rompecabezas de los nueve puntos y su resolución. Fuente: Wikipedia

A lo largo de varios años en el que siempre se imparte este taller siempre se ha encontrado que como mínimo un alumno por grupo de laboratorio (grosso modo una quincena por salón) alcanza la solución al enigma anteriormente planteado después de discurrir un tiempo menor al de diez minutos, permitiendo el docente que el estudiante creativo explique a sus compañeros su brillante solución. La implementación de este sencillo ejercicio también permite al maestro explicar los planteamientos de la Psicología de la Gestalt para así justificar los motivos por las cuales la mayoría de las personas no han podido alcanzar la respuesta correcta, pues desde este paradigma se explica que es por medio de la relación sujeto-objeto donde el individuo extrae los datos más importantes del objeto, incluyendo su perímetro, y así se constituye la información como un todo (Oviedo, 2004). Pues desde la gestáltica ley del cierre "toda información que contribuya a la conformación del concepto de contorno es privilegiada por sobre aquella que no contribuye a darle bordes o límites definidos a los objetos" (Oviedo, 2004, p. 94).

Así pues, el no querer incumplir ese principio de la percepción humana sería la principal razón por la cual el alumno no alcanza la solución al problema puesto que es imposible unir los nueve puntos del rompecabezas sin salir de ese invisible límite que la persona se autoimpuso como una norma interna que no debe ser quebrantada.

\section{La enseñanza de la creatividad en el programa de psicología de la UTB desde las humanidades}

Por medio de la actualización de los desarrollos tecnológicos y el trabajo multidisciplinar de las diferentes facultades de la Universidad
Tecnológica de Bolívar, entendido este concepto como una estrategia pedagógica que implica el diálogo colaborativo de diferentes disciplinas (Van der Linde, 2007), se pretende conseguir que el estudiante mantenga un firme contacto de la realidad social a través de diferentes paradigmas y perspectivas que proporcionan docentes de campos muy diferentes, proceso de suma complejidad (Carvajal, 2010). A pesar de esta dificultad, se fomenta de esta manera el pensamiento crítico y la creatividad gracias (entre otras acciones) a las actividades realizadas por la Coordinación de Humanidades (por medio del apoyo de grupos de lectura, grupos de debate, actuaciones de teatro...).

La enseñanza humanística en la Universidad Tecnológica de Bolívar responde a la intención de formar a estudiantes abiertos a todo tipo de culturas. Es por ello por lo que se ofrece en este centro educativo un repertorio bastante amplio de asignaturas de las áreas de la Filosofía, la Literatura y la Lingüística, la Historia, los Estudios Sociales y Culturales, y el Arte ${ }^{8}$ que puedan ser de interés a todos los alumnos universitarios (indistintamente del pregrado que cursen) por su atractivo intrínseco?. Así pues, dentro del campo de la creatividad, objeto de estudio de nuestro artículo, se pueden destacar dos asignaturas electivas de la Coordinación de Humanidades que en su título especifican la importancia de este concepto:

1. Taller de escritura creativa (área de Literatura y Lingüística).

\section{Fotografía creativa (área de Arte).}

Taller de escritura creativa se asume y comprende como un espacio educativo donde se inician las habilidades creativas estudiantiles en el campo de la escritura. Esta materia se plantea como una serie de actividades lúdicas y gamificadas (Ardila-Muñoz, 2019), las cuales permiten al alumno comprender la realidad literaria como una magnífica herramienta que además de expresar conocimiento también favorece el establecimiento de diálogos críticos. En consecuencia, el estudio de la literatura (yparticularmente la literatura latinoamericana)

8 Las materias del ámbito artístico que se ofertan dentro en la UTB (las cuales consideramos están asociadas con lo creativo) son las siguientes: Dibujo, Pintura, Apreciación del arte, Historia del arte, Fotograffía creativa, Apreciación musical y Foldor colombiano.

9 El alumno de psicología de la UTB debe cursar obligatoriamente dos materias electivas de humanidades durante su plan de estudios para poder graduarse. 
permite acercar al educando las complejas construcciones culturales de su entorno más cercano desde una cosmovisión auto-reflexiva y única (Colomer, 2001). En palabras de Alonso $(2017$, p. 52):

\begin{abstract}
Escribir textos de intención literaria permite al que aprende descubrir racionalmente el proceso de creación artística. El adolescente se acostumbra a trabajar con objetivos su imaginación, a elaborar intenciones y efectos literarios desde temas y argumentos, a profundizar en una técnica de escritura, a presentar textos que deben ser contrastados por los demás, a situar la escritura literaria como resultado de un proceso elaborado, consciente y público.
\end{abstract}

Respecto a Fotografía creativa, es evidente que esta disciplina cumple una función comunicativa de primer nivel dentro de la ya mencionada construcción cultural de las sociedades, pues la fotografía nos permite crear memorias y producir elementos artísticos que ayudan a la interpretación del contexto histórico-social y los modos en los que se desarrollan las personas en sus entornos próximos, una estrategia didáctica-formativa que ha sido minusvalorada por completo (Rodríguez-Ortiz y Montoya-Trujillo, 2020).

Para ello, la materia invita a los estudiantes a plantear discursos críticos (de corte creativo) sobre las problemáticas sociopolíticas y culturales que les rodean por medio de la creación de imágenes fotográficas, como documentos didácticos e históricos que son (Devoto, 2013), que se interpretan, presentan y explican al público universitario en general en exposiciones diseñadas ad hoc para estos propósitos educativos (Figura 2).

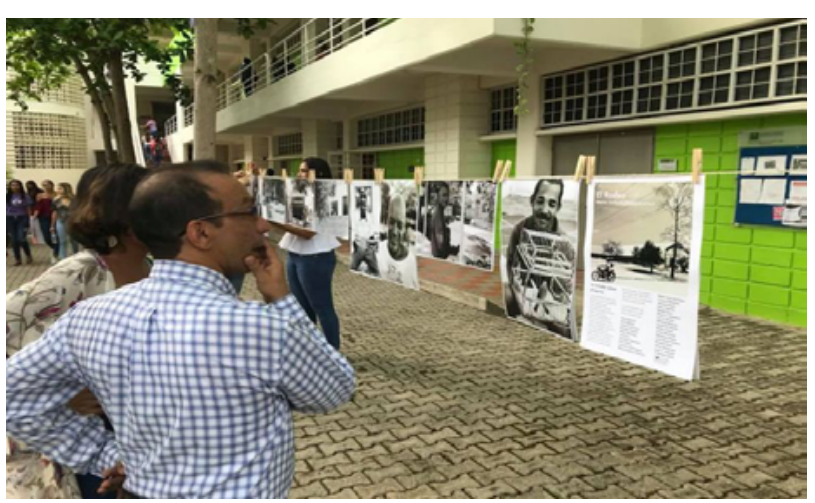

Figura 2. Exposición de fotografías realizadas por estudiantes universitarios en la Muestra Inicial Audiovisual (MIA) en el año 2018. Fuente: Universidad Tecnológica de Bolívar
Los cursos electivos de humanidades son impartidos en clases teóricas en encuentros de dos horas semanales, apoyándose el profesor en presentaciones y trabajos de los propios alumnos universitarios con el objetivo didáctico enfatizar el aspecto práctico de estas materias. Por ende, los jóvenes que cursan estas materias tienen la posibilidad de realizar interpretaciones a piezas literarias y a imágenes artísticas afines con los elementos básicos que componen la escritura y la fotografía respectivamente. El docente busca estimular en sus clases de manera activa las habilidades del pensamiento, la observación comparativa o el análisis crítico, entendidas éstas como aspectos clave de la propia universidad la cual debe estar ligada al desarrollo humanístico (Pérez-Muñoz y Castaño-Calle, 2016).

\section{La enseñanza de la creatividad en el programa de psicología de la UTB desde la economía empresarial}

Dentro de la misión formativa que tienen los programas de pregrado de la UTB, la enseñanza que se imparte en este centro está perfilada para que los egresados de esta institución aporten de forma activa, desde sus conocimientos teóricos y prácticos, insumos para conseguir la mejora económica y social de la región Caribe colombiana ${ }^{10}$. Es por eso por lo que en el plan de estudios del programa de psicología de la UTB se oferta en el quinto semestre la materia obligatoria de Creatividad y emprendimiento, una materia que se vincularía a otras facultades diferentes a la que reporta este programa, tales como la Facultad de Economía y Negocios y la Facultad de Ingeniería (en particular a los programas de pregrado de Administración de Empresas e Ingeniería Industrial). La asignatura está enfocada hacia el sector empresarial pues el estudiante tiene que plantear desde sus inicios una idea innovadora que pueda ser implementada en el ámbito económico como un proyecto de negocio ${ }^{11}$ y

10 Este modelo pedagógico se empezó a elaborar en el año 1998 en la Universidad Tecnológica de Bolívar por el profesor Luis Carlos Arraut, referente local sobre creatividad y emprendimiento (Arraut, Sánchez y Novoa, 2011). La finalidad de este modelo es la capacidad formativa de los estudiantes universitarios a la hora de detectar oportunidades de negocios (Arraut, Amary Duque, 2009).

11 Así la presentación de los mejores modelos de negocio se realiza en un evento denominado Demoday y los ganadores de esta actividad presentan sus proyectos ante los empresarios de la ciudad de Cartagena de Indias en un acto que se denomina Desatiando tiburones. 
así elaborar un marco teórico y práctico en la que se vislumbre las posibilidades reales de estas actividades. Por consiguiente, se busca desarrollar en el estudiante la capacidad creativa, emprendedora y de innovación, con miras a dotarlo de herramientas que le permitan desempeñarse como un emprendedor.

Creatividad y emprendimiento es un curso que fortalece las capacidades y habilidades creativas e innovadoras de los estudiantes donde se brinda metodologías didácticas, que facilitan la aceleración y el inicio de procesos de emprendimiento innovador generando así un valor agregado para diferenciar nuevos modelos de negocio. Esta asignatura hace parte del componente de formación profesional en el área de Gestión Personal (a través del apoyo a la Dirección de Investigación, Emprendimiento e Innovación) y es transversal a las distintas áreas de conocimiento de la Universidad Tecnológica de Bolívar.

El núcleo en el que gira la temática de este curso es el laboratorio El Patio, la principal herramienta de la UTB para el desarrollo de capacidades y habilidades de innovación de estudiantes de pregrado y posgrado de esta universidad (Machado, Huertas-Cardozo y Arraut, 2018) el cual trabaja esta temática desde cuatro fases (Arraut y Gómez, 2017, p. 316):

- Descubrimiento del cliente. Es a su vez la hipótesis de los fundadores iniciales sobre los clientes, el mercado y la solución (producto y/o servicio) con el fin de buscar el encaje entre el problema y la solución.

- Validación de clientes. Prueba si el modelo de negocio resultante es repetible y escalable.

- La creación de clientes. Es la parte inicial de la ejecución. Crea la demanda en los usuarios finales y la dirige a través del canal de ventas para escalar el negocio.

- La creación de empresa. Transición que hace una organización de una start-up a una empresa enfocada a ejecutar un modelo de negocio validado.

Así pues, las ideas innovadoras de esta institución académica se identifican principalmente a través de asignaturas específicas de emprendi- miento, fundamentando su ser en motivar en el alumnado la actitud empresarial (Arraut et al., $2011)$. Esta unidad está conformada por un grupo de investigación ${ }^{12}$ que es dirigido por el Dr. Luis Carlos Arraut. Dentro de las metodologías que manejan estos investigadores destacarían el modelo de clase inversa, estrategia usada para fomentar el pensamiento creativo en contextos universitarios (Al-Zahrani, 2015), y una forma genuina de enseñanza de la creatividad que se elaboró con un sello autónomo y local: el modelo Creinnova (Figura 3).

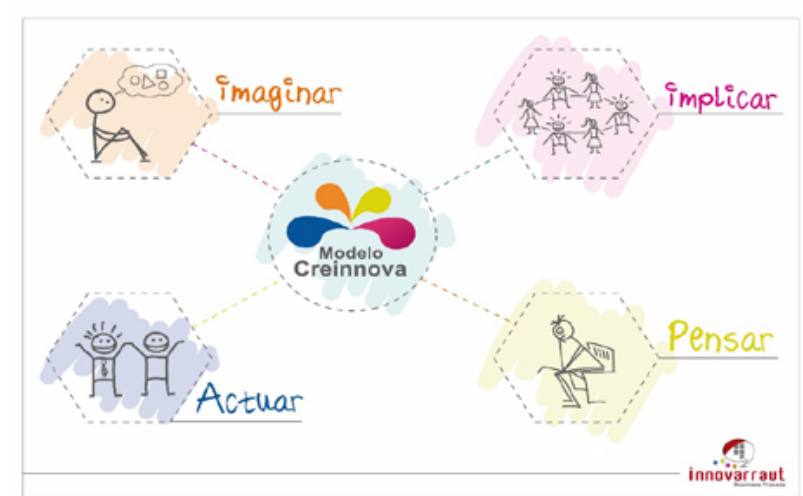

Figura 3. Diagrama del Modelo Creinnova. Fuente: Arraut (2018)

Esta particular forma de enseñar la creatividad se fundamenta en el aprendizaje por desafíos o retos y constaría de cuatro estadios principales: Imaginar, Implicar, Pensar y Actuar. Cada actividad docente se inicia con el lanzamiento de un desafío por parte del profesor para posteriormente tener que construir los estudiantes la solución creativa a ese mismo desafío, debiéndose presentar en público el fruto de su trabajo de manera obligatoria para finalizar con una auto-reflexión final sobre todo este proceso de aprendizaje (Arraut, 2018). Las herramientas que se manejan en este modelo educativo son las siguientes: el pensamiento visual $^{13}$, Wake Up Brain, Lego Serious Play y el Gamestorming (Arraut, 2018). A continuación, pasamos a describir los tres últimos instrumentales citados, todos ellos elementos específicos del área de la innovación y la creatividad (Ovallos, Maldonado y De la Hoz, 2015, p. 101):

12 Grupo de investigación escalafonado por el Ministerio de Ciencias de Colombia en la categońa de B (2020).

13 Témino acuñado por Rudolf Amheim en el año 1969, asumiéndose este tipo de pensamiento como la conexión entre la percepción y la concepción a través de la habilidad de ver las formas visuales como imágenes (Púñez, 2017). 
- Wake Up Brain: conjunto de herramientas y actividades [diseñadas por Guillermo Solano desde el año 2012] que llevan a encontrar conceptos innovadores de alto potencial, usando una aproximación de pensamiento ambidiestro.

- Lego Serious Play [autoría Play And Build, año 2012]: metodología que diseña competencias que permitan innovar y mejorar el desempeño del personal de las organizaciones.

- Gamestorming [autor clave: David Gris, año 2010]: metodología basada en una serie de juegos para potenciar la gestión de la innovación en las organizaciones.

\section{DISCUSIÓN Y CONCLUSIONES}

Indicándose todo lo anterior, concluimos que la Universidad Tecnológica de Bolívar ofrece a sus estudiantes de psicología el adquirir conocimientos relacionados con la creatividad desde paradigmas académicos completamente diferentes (psicología cognitiva, humanidades y economía empresarial) y desde varios enfoques metodológicos, un aspecto que consideramos diferencial frente a otras instituciones de educación superior de la región. Si bien, esta fortaleza pedagógica que se ha identificado no es óbice para que se hayan detectado también algunas debilidades durante los últimos años y que indicamos a continuación de forma esquematizada:

1. Imposibilidad de un estudiante en dar todos los procesos psicológicos básicos en contraste con la presencia en la malla curricular de psicología de otras materias ajenas a esta disciplina.

2. Excesivo uso de términos ambiguos y manejo de lenguaje acientífico basado en respuestas circulares (no vinculadas a las humanidades) para explicar una característica psicológica de valencia favorable como el ser creativo/a. Un aspecto que vinculamos a las limitaciones inherentes del paradigma de la psicología positiva (Fernández-Ríos y Vilariño, 2016) frente (por ejemplo) al número de patentes registradas en la universidad como sinónimo de un indicador medible, cuantificable y observable de creatividad e innovación.
3. Presencia de sesgos en diversas prácticas sociales que muestran "a otros grupos sociales como poco proactivos, poco inteligentes e incapaces de contribuir al desarrollo social de la región y el país" (Ararat, 2010, p. 31).

4. Rechazo a los enfoques mercantilistas dirigidos a la creación de micro-empresas cuando la mayoría de psicólogos que trabajan por libre desempeñan su trabajo en un rígido marco legal establecido por las autoridades sanitarias.

5. Desinterés de muchos alumnos por las materias electivas de humanidades ${ }^{14}$, como tal, al considerarlas asignaturas secundarias (Rodríguez-Ortiz y Montoya-Trujillo, 2020) o únicamente cursos que per se, deben aumentar el promedio académico.

Ante estos retos al mantenimiento de la calidad educativa que han sido detectados (entre otros factores no vinculantes a la temática de este texto), recientemente el programa de psicología de la UTB ha modificado su malla curricular con el fin de adaptarse a las nuevas necesidades de las futuras generaciones, pasando el plan de curso de ocho semestres a nueve (a diferencia de la reducción de los pregrados en España). Una variación aprobada por las pertinentes autoridades educativas colombianas y que próximamente entrará en vigor. Los cambios más significativos que atañen a las críticas señaladas en este mismo apartado serían los siguientes:

1. Reestructuración de las materias de psicología cognitiva para que los alumnos den todos los procesos psicológicos básicos, sin posibilidad de elección por parte del alumnado.

2. Aumento de las electivas de humanidades (de dos a tres) en el pensum de la carrera. Es primordial para la UTB darles valor a los conocimientos humanísticos en base al modelo pedagógico, la misión y el objetivo de esta institución como universidad que es (Pérez-Muñoz y Castaño-Calle, 2016) y así se debe transmitir a la comunidad estudiantil para su mejor comprensión y entendimiento.

14 Problema que se ve agravado en los estudiantes de la Facultad de Ingenieńa al asumir emóneamente muchos de ellos que esta área del saber carece de sentido para su formación académica. 
3. Modificación en el formato de todos los sílabos de las materias de la carrera asignándose a los planes de curso un formato por semanas con su respectiva bibliografía de referencia para cada actividad pedagógica que el docente planifique.

En definitiva, entendemos que con estas mejoras que se implementarán en un futuro, el programa de psicología de la UTB mantendrá la enseñanza de la creatividad desde una perspectiva multidisciplinar reforzando aspectos específicos de su malla sin alterar la ventaja competitiva resultado del primer punto señalado. Recalcamos la idea de que un psicólogo de esta universidad que haya adquirido perspectivas tan dispares en un concepto tan relevante como la creatividad es algo positivo que generará un retorno positivo en estos recién egresados cuando se incorporen al mercado laboral al haberse formado estos jóvenes de manera integral. Por ende, La UTB aspira a formar psicólogos con altos niveles de calidad académica que tengan un pleno desarrollo en las competencias propias del saber y el saber-hacer de esta específica profesión, pero nunca olvidando la formación del ser.

Es por ello por lo que asumimos que la diversidad de la enseñanza de la creatividad es algo fundamental y se ha creído pertinente el presentar a la comunidad esta experiencia pedagógica originaria de una humilde universidad privada de Colombia.

\section{REFERENCIAS}

Alessandroni, N. (2017). Imaginación, creatividad y fantasía en Lev S. Vygotski: una aproximación a su enfoque sociocultural. Actualidades en Psicología, 31 (122), 4560. doi: 10.15517/ap.v31i122.26843

Alonso, D. (2016). El desarrollo de la autodeterminación a través del proceso creativo de las personas con discapacidad intelectual. Artseduca, 15, 70-95.

Alonso, F. (2017). Didáctica de la escritura creativa. Tarbiya. Revista de Investigación e Innovación Educativa, 28, 51-66.

Al-Zahrani, A.M. (2015). From passive to active: The impact of the flipped classroom through social learning platforms on higher education students' creative thinking. British Journal of Educational Technology, 46(6), 1133-1148. doi: $10.1111 /$ bjet. 12353

Amabile, T. (2019). The Art of (Creative) Thought: Graham Wallas on the Creative Process. En V.P. GI $\square$ veanu (Ed.), The Creativity Reader (pp. 15-32). Oxford: Oxford University Press.

Ararat, J.A. (2010). La ideología del emprendimiento. Una mirada desde el análisis crítico del discurso. AD-minister, 17, 5-33.

Ardila-Muñoz, J.Y. (2019). Supuestos teóricos para la gamificación de la educación superior. Magis. Revista Internacional de Investigación en Educación, 12(24), 71-84. doi: 10.11144/Javeriana.m12-24.stge

Arraut, L.C. (2018). Modelo Creinnova: propuesta metodológica de enseñanza para la innovación basada en desafíos. Encuentro Internacional de Educación en Ingeniería ACOFI 2018.

Arraut, L.C., Amar, P. y Duque, J. (2009). Hacia un modelo de emprendimiento de innovación abierta totalmente responsable: caso Universidad Tecnológica de Bolívar en Colombia. TEC Empresarial, 3(1-2), 33-44.

Arraut, L.C. y Gómez, E. (2017). Prototipado rápido para desarrollo de conceptos en ideas de negocio innovadoras. En J. Bernal et al. (Ed.), Hagamos visible el desarrollo científico e innovador de la UTB. Memorias Simposio de Investigación 2017 (pp. 314 322). Cartagena de Indias: Editorial Universidad Tecnológica de Bolívar.

Arraut, L.C., Sánchez, H. y Novoa, K. (2011). Impacto del programa emprendedor en la Universidad Tecnológica de Bolívar mediante un análisis a través de SPSS. Revista de la Escuela de Administración de Negocios, 71, 184-195.

Carvajal, Y. (2010). Interdisciplinariedad: desafío para la educación superior y la investigación. Revista Luna Azul, 31 , 156-169.

Cedeño-Sempértegui, M.L. (2015). La acción dinamizadora de los Syllabus: una experiencia en construcción. Yachana. Revista Científica, 4(Ed. Especial), 129-135. 
Colomer, T. (2001). La enseñanza de la literatura como construcción del sentido. Lectura y Vida. Revista Latinoamericana de Lectura, 22(1), 6-23

Dahl, S. (2007). Turnitin $®$ : The student perspective on using plagiarism detection software. Active Learning in $\mathrm{Hi}$ gher Education, 8(2), 173-191. doi: $10.1177 / 1469787407074110$

D’Angelo, O. (1999). Proyección desde Vigotsky a la construcción de la persona y la sociedad creativas. Revista Cubana de Psicología, 16(2).

De la Torre, S. y Violant, V. (2002) Estrategias creativas en la enseñanza universitaria. Una investigación con metodología de desarroIlo. Creatividad y Sociedad, 3, 21-38.

Devoto, E. (2013). La imagen como documento histórico-didáctico: algunas reflexiones a partir de la fotografía. Revista de Educación, $6,73-94$

Elisondo, R., Donolo, D. y Rinaudo, M.C. (2009). Ocasiones para la creatividad en contextos de educación superior. Red U. Revista de Docencia Universitaria, 7(4).

Fernández-Ríos, L. y Vilariño, M. (2016). Mitos de la psicología positiva: maniobras engañosas y pseudociencia. Papeles del Psicólogo, 37(2), 134-142.

Fleith, D. S. (2019). The role of creativity in graduate education according to students and professors. Estudos de Psicologia (Campinas), 36. doi: 10.1590/1982. 0275201936 e180045

Guilford, J.P. (1956). The structure of intellect. Psychological Bulletin, 53(4). 267-293. doi: $\underline{10.1037 / \mathrm{h} 0040755}$

Gutiérrez-Braojos, C., Salmeron-Vilchez, P., Martin-Romera, A. y Salmerón Pérez, $H$. (2013). Efectos directos e indirectos entre estilos de pensamiento, estrategias metacognitivas y creatividad en estudiantes universitarios. Anales de Psicología, 29(1), 159 170. doi: 10.6018/analesps.29.1.124651

H. del Amo, O. (2016). Impacto de materias artísticas en currículos de grado. Artseduca, 14, 76-97.
Huidobro, T. (2004). Una definición de la creatividad a través del estudio de 24 autores seleccionados. Tesis doctoral: Universidad Complutense de Madrid.

Jelle, B.P. (2017). Reviewing the Learning Process through Creative Puzzle Solving. Creative Education, 8, 2009-2035. doi: h10.4236/ce.2017.813137

Kettler, T., Lamb, K.N., Willerson, A., y Mullet, D.R. (2018). Teachers' Perceptions of Creativity in the Classroom. Creativity Research Journal, 30(2), 164-171. doi: 10.1080/10400419.2018.1446503

López-Martínez, O. y Martín-Brufau, R. (2010). Estilos de pensamiento y creatividad. Anales de Psicología, 26(2), 254-258.

Machado, S.E., Huertas-Cardozo, N.C., y Arraut, L.C. (2018). Laboratorio El Patio, impacto de la inversión en gestión de la innovación en los entornos de la Universidad Tecnológica de Bolívar. Revista Vinculategica Efan, 3(2), 223-230.

Maslow, A.H. (1968). Toward a psychology of being. Nueva York: D. Van Norstrand.

Nuha, M.A., Waluya, S.B., y Junaedi, I. (2018). Mathematical Creative Process Wallas Model in Students Problem Posing with Lesson Study Approach. International Journal of Instruction, $17(2)$, 527-538. doi: 10.12973/ iii.2018.11236a

Ovallos, D.A., De la Hoz, S.M. y Maldonado, D.J. (2015). Creatividad, innovación y emprendimiento en la formación de ingenieros en Colombia. Un estudio prospectivo. Revista Educación en Ingeniería, 10(19), 90104.

Oviedo, G.L. (2004). La definición del concepto de percepción en psicología con base en la teoría Gestalt. Revista de Estudios Sociales, 18, 89-96. doi: 10.7440/ res18.2004.08

Pérez-Muñoz, S. y Castaño-Calle, R. (2016). Funciones de la Universidad en el siglo XXI: humanística, básica e integral. Revista Electrónica Interuniversitaria de Formación del Profesorado, 19(1), 191-199.

Púñez, N. (2017). El Pensamiento visual: una propuesta didáctica para pensar y crear. 
Horizonte de la Ciencia, 7(12), 161-177.

Rodríguez-Ortiz, A.M. y Montoya-Trujillo, B.M. (2020). La fotografía como estrategia para formar en ciudadanía. Jangwa Pana, 19(1), 150-178. doi: 10.21676/16574923.3359

Rogers, C. (1975). Libertad y creatividad en la educación. Buenos Aires: Paidós.

Romo, M. (1986). Treinta y cinco años del pensamiento divergente: teoría de la creatividad de Guilford. Estudios de Psicología, 7(27-28), 175-192. doi: $\underline{10.1080 / 02109395.1986 .10821474}$

Romo, M., Alfonso-Benlliure, V. y SánchezRuiz, M.J. (2016). El test de creatividad infantil (TCl): evaluando la creatividad mediante una tarea de encontrar problemas. Psicología Educativa, 22(2), 93-101. doi: 10.1016/i.pse.2016.01.005

Rosen, K.H. (2003). Discrete Mathematics and Its Applications. Nueva York: McGraw Hill Education.

Sadler-Smith, E. (2015). Wallas' FourStage Model of the Creative Process: More Than Meets the Eye? Creativity Research Journal, 27(4), 342-352. doi: $\underline{10.1080 / 10400419.2015 .1087277}$

Snyder, H.T., Hammond, J.A., Grohman, M.G. y Katz-Buonincontro, J. (2019). Creativity measurement in undergraduate students from 1984-2013: A systematic review. Psychology of Aesthetics, Creativity, and the Arts, 13(2), 133-143. doi: 10.1037/ aca0000228

Soh, K.C. (2000) Indexing creativity fostering teacher behaviour: a preliminary validation study. Journal of Creative Behaviour, $34(2), \quad 118-134$. doi: 10.1002/i.21626057.2000.tb01205.x

Tamayo, M. (2004). El proceso de la investigación científica. Ciudad de México: Limusa.

Van der Linde, G. (2007). ¿̇Por qué es importante la interdisciplinariedad en la educación superior? Cuadernos de Pedagogía Universitaria, 4(8), $11-13$.

Vázquez-Miraz, P., Rentería, C., Martínez, M.J. y Zapata, K. (2020). Principales dificultades del alumnado universitario novel a la hora de elaborar un texto científico. Tejuelo, 32, 117-146.

Wallas, G. (1926). The art of thought. Londres: Jonathan Cape.

Zhu, C. y Zhang, L. (2011). Thinking styles and conceptions of creativity among university students. Educational Psychology, 37 (3), 361-375. doi: $10.1080 / 01443410.2011 .557044$ 